\title{
INDICATIONS FOR DISTAL RADIOULNAR ARTHROPLASTY: REPORT ON THREE CLINICAL CASES
}

Cláudia Santos ${ }^{1}$, Alexandre Pereira ${ }^{2}$, Marco Sousa $^{2}$, Miguel Trigeuiros $^{2}$, César Silva $^{2}$

\section{ABSTRACT}

Distal radioulnar arthroplasty is an attractive solution for treating various pathological conditions of the distal radioulnar joint because it allows restoration of stability, load transmission and function. The main indications are: radioulnar impingement after partial or complete resection of the distal ulna; and degenerative, inflammatory or post-traumatic arthritis of the distal radioulnar joint. The authors present three clinical cases of distal radioulnar pathological conditions: two patients with post-traumatic sequelae and one case of distal radioulnar impingement after a Sauvé-Kapandji operation. The three cases were treated surgically with a metallic prosthesis to replace the distal ulna (First Choice - Ascension ${ }^{\circledR}$ ). The first two were treated with a resurfacing prosthesis and the last one with a modular prosthesis. All of the patients had achieved pain relief and increased movement of the distal radioulnar joint after one year of postoperative follow-up.

Keywords - Arthroplasty; Wrist/pathology; Wrist/surgery.

\section{INTRODUCTION}

Distal radioulnar arthrosis is relatively common and gives rise to incapacitating pain, with diminution of wrist strength and stiffness of pronosupination. The main causes are trauma, inflammation and distal radioulnar instability.

The classical surgical procedures used for treating arthrosis of the distal radioulnar joint depend in some way on total or partial excision of the distal ulna, such as the Darrach, Bower and Sauvé-Kapandji operations. However, these procedures do not allow restoration of the anatomy, and there is a real risk of producing symptomatic instability. This could give rise to a disorder that is more severe or incapacitating than the original condition.

Distal radioulnar arthroplasty with an implant is an attractive treatment for pathological conditions of the distal radioulnar joint, since this makes it possible to eliminate the pain and restore the anatomy, while maintaining the alignment, forearm function and stability of the distal radioulnar joint.

\section{CLINICAL CASES}

\section{First clinical case:}

The patient was a 50-year-old woman who had suffered a fall from her own height that resulted in trauma to the left wrist. At that time, she sought the emergency services, where she was immobilized with an elastic restraint. Because the pain continued and the range of motion of the left wrist joint diminished, magnetic resonance was performed. The report from this examination showed that the triangular fibrocartilage complex was injured. The patient then underwent wrist arthroscopy with regularization of the fibrocartilage. Durante the follow-up, she did not recover from the pain and the limitation of mobility, which was

1 - Resident Physician in the Orthopedics Service, Porto Hospital Center, Porto, Portugal.

2 - Attending Physicians in the Orthopedics Service, Porto Hospital Center, Porto, Portugal.

Work performed at the Orthopedics Service, Alto Minho Local Healthcare Unit, EPE, Viana do Castelo, Portugal.

Correspondence: Serviço de Ortopedia - Centro Hospitalar do Porto, Portugal - Largo Professor Abel Salazar - 4099-001. E-mail: claudsantos@hotmail.com

Work received for publication: January 12, 2010; accepted for publication: July 19, 2010. 
essentially pronosupination. On radiological examination, degenerative abnormalities of distal radioulnar arthrosis were seen (Figure 1). The patient underwent surgical treatment with implantation of a resurfacing prosthesis to replace the distal ulna (Figure 2).

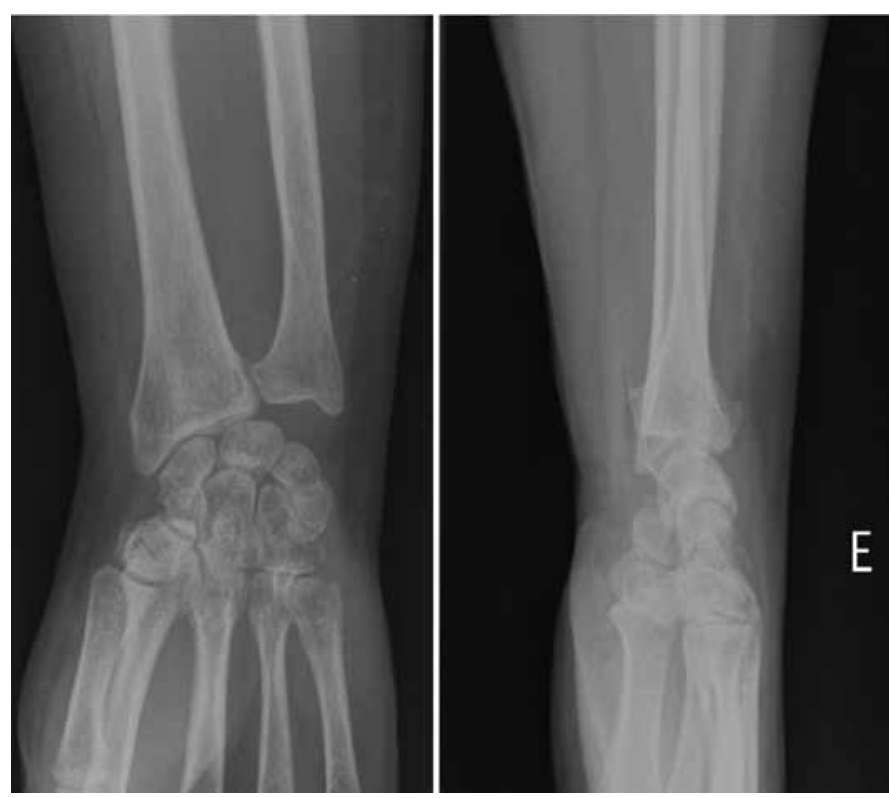

Figure 1 - First clinical case before the operation: showing degenerative abnormalities of the distal radioulnar joint.
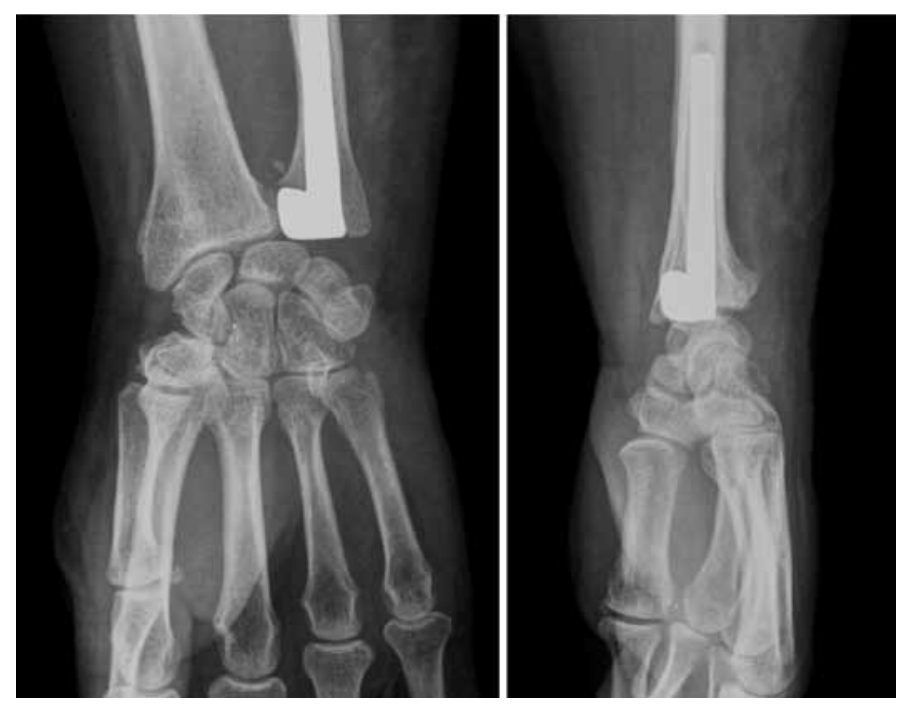

Figure 2 - First clinical case after the operation: the patient underwent resurfacing distal arthroplasty of the ulna.

\section{Second clinical case:}

The patient was a 31-year-old woman with a history of trauma to her left wrist, which had evolved to pseudarthrosis of the ulnar styloid apophysis, with degenerative abnormalities of the distal radioulnar joint (Figure 3). Because the patient's pain continued (essentially on pronosupination) and her capacity to grasp and lift objects was diminished, she was treated with implantation of a resurfacing prosthesis for the distal ulna (Figure 4).
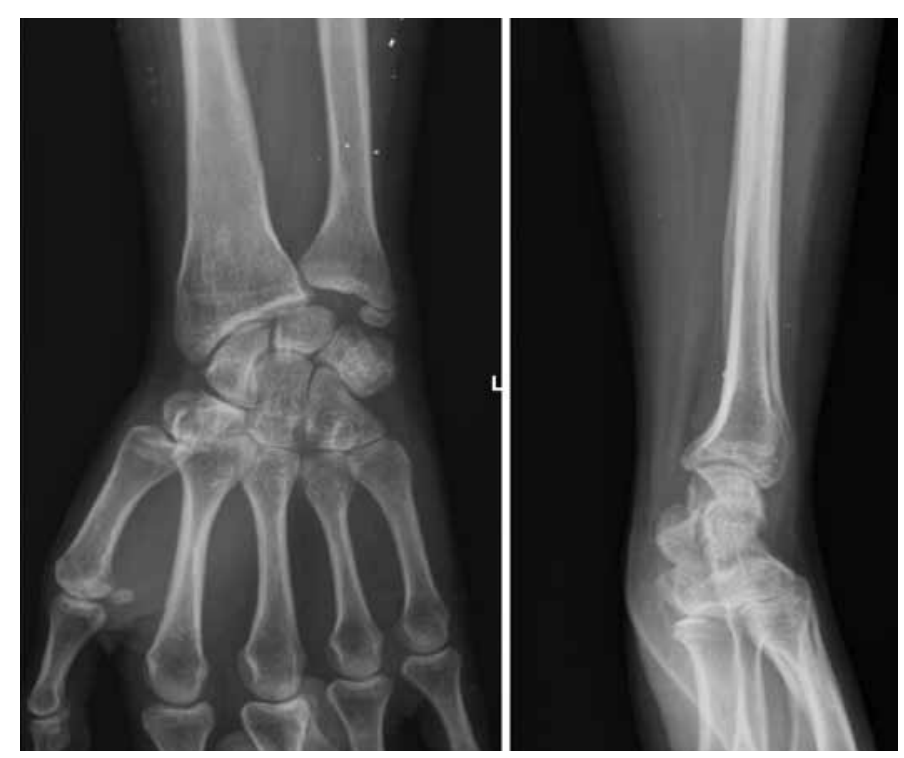

Figure 3 - Second clinical case before the operation: pseudarthrosis of the ulnar apophysis and degenerative abnormalities of the distal radioulnar joint.
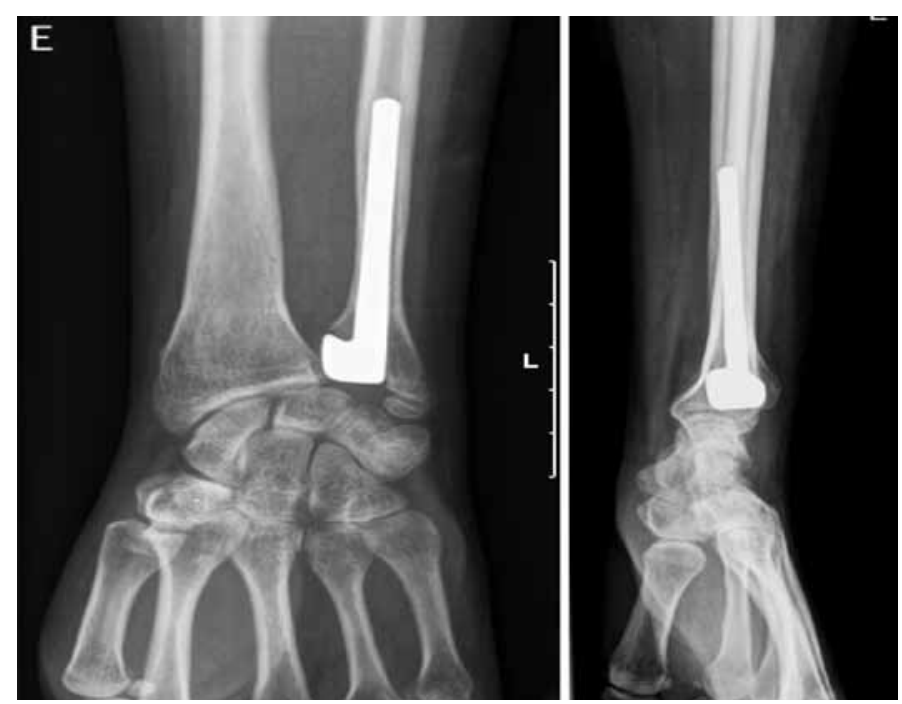

Figure 4 - Third clinical case after the operation: resurfacing distal arthroplasty of the ulna.

\section{Third clinical case:}

The patient was a 33-year-old woman who suffered trauma to the right wrist with distal radioulnar incongruence and underwent the Sauvé-Kapandji operation. During the follow-up, pain on pronosupination was observed, along with a sensation of prominence of the 
proximal stump of the ulna. Radiologically, indirect signs of distal radioulnar impingement syndrome were observed (Figure 5). The patient underwent modular distal radioulnar arthroplasty (Figure 6).

After one years of follow-up, the pain was assessed using a visual scale; the grasping muscle strength was determined using a Jamar ${ }^{\circledR}$ hand dynamometer (5030J1); and the pronosupination joint range of motion was measured. The results were compared with the findings from the preoperative consultation. For all three patients, improvements in the three values on the visual pain scale were registered. With regard to grasping strength, the preoperative mean was 6.5 $\mathrm{kg}$ and the postoperative mean was $8.3 \mathrm{~kg}$. The mean pronosupination range of motion before the operation was $30^{\circ}-5^{\circ}$ and it was $65^{\circ}-50^{\circ}$ after the operation.
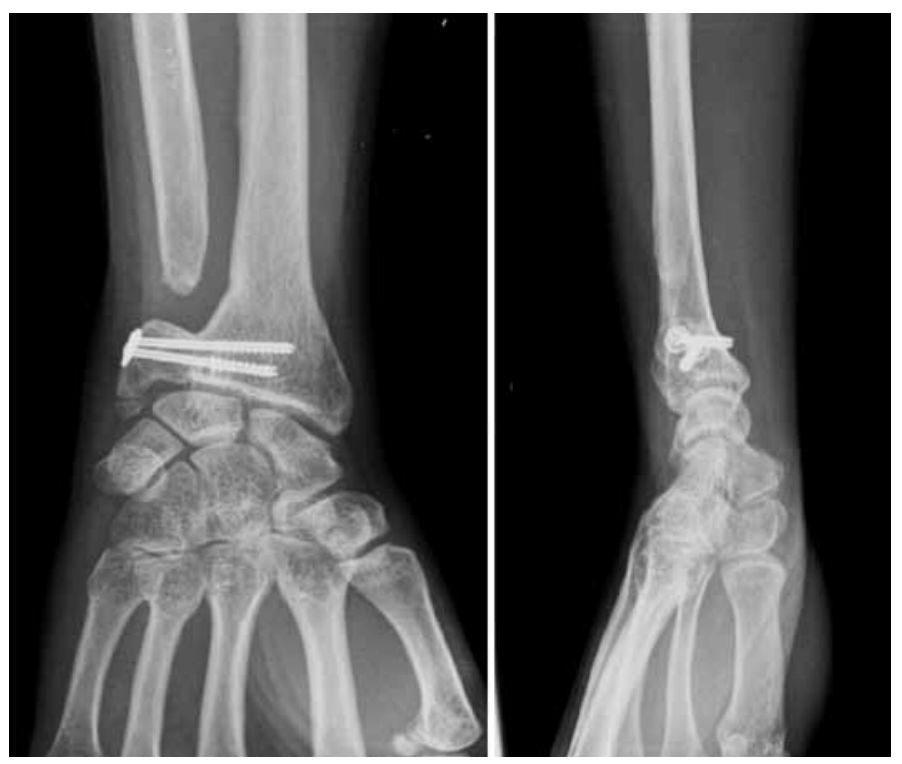

Figure 5 - Third clinical case before the operation: patient with sequelae from the Sauvé-Kapandji operation, with distal radiouInar impingement syndrome.

\section{DISCUSSION}

The main indications for distal radioulnar arthroplasty with an implant are distal radioulnar arthrosis and radioulnar impingement after distal recession of the ulna ${ }^{(1-4)}$.

Several types of implants have been described. Swanson was the pioneer in designing a silicone prosthesis, although this was rapidly abandoned because it led to recurrence of the symptoms, failure of the material and synovitis ${ }^{(1)}$. Subsequently, in 2000, Van
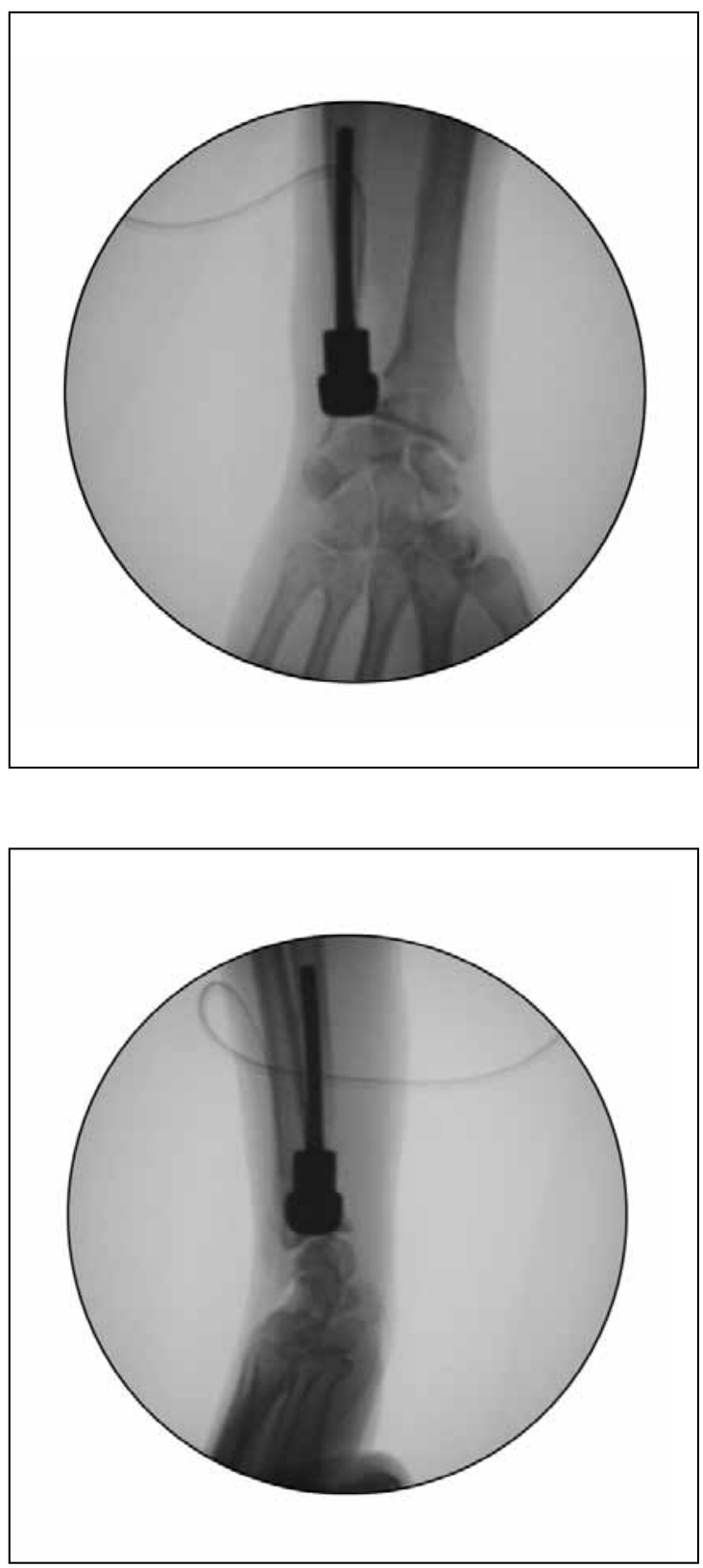

Figure 6 - Third clinical case: modular distal radioulnar arthroplasty.

Schoonhoven et $\mathrm{al}^{(4)}$ published their experience from treating 23 cases of chronic instability of the distal radioulnar joint through implantation of ceramic and a titanium rod. In 2005, Scheker et al ${ }^{(3)}$ also introduced a ball-and-socket prosthesis to totally replace the distal radioulnar joint, which theoretically made it possible to resolve complex cases of bone and ligament loss. More recently, the concept of modular metallic prostheses emerged: these allow better adaptation of the implant and reinsertion of soft tissues at the metallic head, in order to promote stability ${ }^{(2)}$. 
Biomechanical studies have demonstrated that the implants enable restoration of the normal kinetics of the distal radioulnar joint, thus allowing instabilities and impingement to be resolved ${ }^{(2,5)}$. However, there are some technical limitations: reinsertion of the soft tissues at the metallic head may be difficult in patients who have undergone several surgical interventions; and to improve the adaptation of the implant, it may be necessary to remodel and deepen the sigmoid notch of the radius. Nonetheless, distal radioulnar arthroplasty seems to be an effective and predictable solution, with less morbidity than in other surgical options for treating radioulnar arthrosis and impingement. However, further long-term studies will be needed in order to completely assess its usefulness.

\section{REFERENCES}

1. Berg, E. Indications for and results with the Swanson distal ulnar prothesis. South Med J. 1976 , 69(7):858-61.

2. Berger RA, Cooney WP 3rd. Use of an ulnar head endoprosthesis for treatment of an unstable distal ulnar resection: review of mechanics, indications, and surgical technique. Hand Clin. 2005;21(4):603-20.
3. Scheker LR, Babb BA, Killion PE. Distal ulnar prosthetic replacement. Orthop Clin North Am 2001;32(2):365-76.

4. Van Schoonhoven J, Herbert TJ, Fernandez DL, Prommersberger KJ, Krimmer H. [ulnar head prosthesis]. Orthopöde 2003;32(2):809-15.

5. Bell, MJ, Hill RJ, McMurty RY. Ulnar impingement syndrome. J Bone Joint Surg. 1985;67(1):126-9. 